\title{
Somatostatin receptors differentially affect spontaneous epileptiform activity in mouse hippocampal slices
}

\author{
Maurizio Cammalleri, ${ }^{1}$ Davide Cervia, ${ }^{1}$ Daniel Langenegger, ${ }^{2}$ Yanqiang Liu, ${ }^{3}$ Massimo Dal Monte, ${ }^{1}$ Daniel Hoyer $^{2}$ and \\ Paola Bagnoli ${ }^{1}$ \\ ${ }^{1}$ Dipartimento di Fisiologia e Biochimica 'G. Moruzzi', Università di Pisa, 56127 Pisa, Italy \\ ${ }^{2}$ Neuroscience Research, Novartis Institutes for Biomedical Research, Novartis Pharma AG, Basel, Switzerland \\ ${ }^{3}$ College of Life Sciences, Nankai University, Tianjin, China
}

Keywords: epilepsy, extracellular recording, hippocampus, knockout mice, somatostatin analogues

\begin{abstract}
Somatostatin-14 [somatotropin release-inhibiting factor (SRIF)] reduces hippocampal epileptiform activity but the contribution of its specific receptors $\left(s t_{1-5}\right)$ is poorly understood. We have focused on the role of $s t_{1}$ and $s_{2} t_{2}$ in mediating SRIF modulation of epilepsy using hippocampal slices of wild-type (WT) and $s t_{1}$ or $s_{2} t_{2}$ knockout (KO) mice. Recordings of epileptiform discharge induced by $\mathrm{Mg}^{2+}$-free medium with 4-aminopyridine were performed from the CA3 region before and after the application of SRIF compounds. In WT mice, SRIF and the sst $_{1}$ agonist $\mathrm{CH}-275$ reduce epilepsy whereas sst ${ }_{1}$ blockade with its antagonist SRA-880 increases the bursting discharge. Activation of $\mathrm{sst}_{2}$ does not affect the bursting frequency unless its agonist octreotide is applied with SRA-880, indicating that sst ${ }_{1}$ masks sst ${ }_{2}$-mediated modulation of epilepsy. In sst ${ }_{1} \mathrm{KO}$ mice: (i) the bursting frequency is lower than in WT; (ii) SRIF, CH-275 and SRA-880 are ineffective on epilepsy and (iii) octreotide is also devoid of effects, whereas blockade of sst 2 with the antagonist D-Tyr ${ }^{8}$ Cyn 154806 increases the bursting frequency. In sst ${ }_{2} \mathrm{KO}$ mice, the SRIF ligand effects are similar to those in WT. In the whole hippocampus of $s s t_{1} \mathrm{KO}$ mice, sst $_{2} \mathrm{mRNA}$, protein and binding are higher than in WT and reverse transcription-

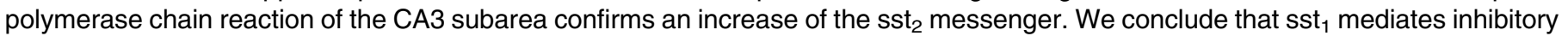
actions of SRIF and that interactions between $\mathrm{sst}_{1}$ and $\mathrm{sst}_{2}$ may prevent $\mathrm{sst}_{2}$ modulation of epilepsy. We suggest that, in sst ${ }_{1} \mathrm{KO}$ mice, activation of over-expressed $s_{2} t_{2}$ reduces the bursting frequency, indicating that $s_{2} t_{2}$ density represents the rate-limiting factor for sst $_{2}$-mediated modulation of epilepsy.
\end{abstract}

\section{Introduction}

Somatostatin-14 [somatotropin release-inhibiting factor (SRIF)] is a neuropeptide widely distributed in different brain regions, including the hippocampal formation (Binaschi et al., 2003). SRIF actions are mediated by five receptor subtypes $\left(\mathrm{sst}_{1-5}\right)$. In the rodent brain, receptor binding studies and immunological analysis have shown a widespread but selective distribution of distinct SRIF receptors (Schulz et al., 2000; Weckbecker et al., 2003). SRIF receptors are coupled to $\mathrm{G}$ proteins and trigger multiple transmembrane signalling systems (Lahlou et al., 2004) mediating different functions of SRIF receptors which also depend on their possible interactions. For example, in recombinant systems, $\mathrm{sst}_{2 / 3}$ and $\mathrm{sst}_{1 / 5}$ receptor interactions have been demonstrated (Rocheville et al., 2000; Pfeiffer et al., 2001). However, little is known about native systems. In particular, functional interactions have been suggested between $\mathrm{sst}_{2}$ and $\mathrm{sst}_{5}$ receptors in rat tumour somatotrophs (Cervia et al., 2003c). In addition, $\mathrm{sst}_{2}$ and $\mathrm{sst}_{4}$ receptors appear to be functionally coupled in the mouse hippocampus (Moneta et al., 2002). Finally, interactions between $\mathrm{sst}_{1}$ and $\mathrm{sst}_{2}$ receptors have recently been suggested in the mouse retina (Pavan et al., 2004).

At the neuronal level, SRIF is involved in multiple functions, including control of excitatory neurotransmission. In the rat hippocampus, SRIF inhibits neuronal excitability by affecting $\mathrm{Ca}^{2+}$ and $\mathrm{K}^{+}$

Correspondence: Professor P. Bagnoli, as above.

E-mail: pbagnoli@dfb.unipi.it

Received 21 June 2004, revised 8 September 2004, accepted 13 September 2004 conductances (Boehm \& Betz, 1997; Schweitzer et al., 1998; Baraban $\&$ Tallent, 2004). In addition, there is evidence indicating that SRIF, possibly released by SRIF-containing interneurones participating in the lateral inhibitory circuits in the dentate gyrus (Buckmaster et al., 2002; Kobayashi \& Buckmaster, 2003), reduces epileptiform activity in both CA1 and CA3 hippocampal regions (Tallent \& Siggins, 1997, 1999; Vezzani \& Hoyer, 1999; Baraban \& Tallent, 2004). These observations suggest that SRIF plays a role in seizure control and therefore that it may have an importance as a potential therapeutic agent for temporal lobe epilepsy (Vezzani \& Hoyer, 1999; Binaschi et al., 2003).

Information on the precise contribution of each SRIF receptor subtype on the SRIF-induced inhibition of epileptiform activity is still limited. Of the five SRIF receptors, the sst $_{2}$ receptor is the candidate likely to mediate the anticonvulsant effects of SRIF in the hippocampus and entorhinal cortex of rats (Vezzani \& Hoyer, 1999). Previous findings, however, have demonstrated that, in mice, these receptors do not mediate inhibitory effects of SRIF on seizure susceptibility and hippocampal excitatory neurotransmission. Rather, excitatory actions of $\mathrm{sst}_{4}$ receptors have been reported (Moneta et al., 2002).

No information is available on the $\mathrm{sst}_{1}$ receptor function in hippocampal physiology. In the rodent hippocampus, for instance, the expression of $\mathrm{sst}_{1}$ receptors has not been conclusively demonstrated using either immunohistochemistry or radioligand binding (Hervieu \& Emson, 1998; Schulz et al., 2000; Videau et al., 2003), whereas previous studies have suggested that hippocampal neurones may express sst $_{1}$ receptor mRNA (Perez et al., 1994; Hannon et al., 2002b). 